\title{
Book review: Creative Methods for Human Geographers
}

\author{
Jeannine Wintzer \\ Geographisches Institut, Universität Bern, Bern, Schweiz \\ Correspondence: Jeannine Wintzer (jeannine.wintzer@giub.unibe.ch) \\ Published: 22 July 2021
}

Von Benzon, N., Holton, M., Wilkinson, C., and Wilkinson, S. (Eds.): Creative methods for human geographers, Sage, London, 432 pp., ISBN 978-1-5264-9697-3, EUR 37.40, 2021 .

Qualitative Methoden gehören seit mehr als 30 Jahren zum methodischen Repertoire der Geographie. Mit dem Ziel, verstehende Zugänge zur sozialräumlichen Wirklichkeit zu schaffen, griffen Geograph*innen in den Anfangsjahren qualitativ orientierter Forschung auf etablierte Methoden der Sozialwissenschaft zurück. Beginnend mit der Erhebung und Auswertung qualitativer Daten durch Interviewformen und inhaltsanalytische Verfahren, bedienten sich Geograph*innen immer mehr des sich seit den 1980er-Jahren differenzierenden Methodenkoffers der Sozialwissenschaft. Dieser Transfer war von dem Prinzip getragen, dass die jeweilige Methode eins zu eins im Sinne des in sich stringenten Forschungsdesigns zu übernehmen sei. Die zunehmende Differenzierung der sozialräumlichen Welt liess an dieser starren Methodenanwendung Zweifel aufkommen; zunehmend waren und sind methodische Ansätze gefragt, die je nach Forschungsziel und -kontext flexibel und kreativ anwendbar sind. Nicht die Methode an sich, sondern deren Anwendungskontext und damit deren Anpassung an die Bedürfnisse innerhalb des geographischen Forschungsprozesses sind für die Auswahl der Methode entscheidend.

An diesem Punkt beginnt die Arbeit des Sammelbandes Creative methods for human geographers. Schon der Titel produziert hohe Erwartungen, die umfänglich erfüllt werden. Creative ist nur bedingt ein Labeling der vorgestellten methodischen Zugänge; vielmehr wird damit die Position vertreten, dass kreative Lösungen nicht einem Mangel an Planung innerhalb von Forschungsprozessen entspringen, sondern grundlegender Bestandteil von Wissenschaft sind. Damit spiegeln die Beiträge einen Perspektivwechsel innerhalb der Sozialforschung wider: Nicht die strikte Durchfüh- rung einer Methode, sondern die kreative Anpassung an das zu Untersuchende und die damit einhergehende innovative (Neu-)Anwendung von Methoden steht im Mittelpunkt des Sammelbandes. Dies verändert auch die Forschungspraktiken qualitativ orientierter Geographien: Etablierte Methoden werden für die Geographie nicht nachgestellt, sondern die Beiträge schaffen Raum für die eigens von und für die Geographie geschaffenen methodischen Zugänge zur sozialräumlichen Welt. Damit gelingt es den Beiträgen, sich dem Dualismus zwischen qualitativer und quantitativer Forschung zumindest einmal sprachlich zu entziehen. Als Ersatz wird von den Autor*innen Kreativität als ein Mittel angeboten, Neues zu erkunden und damit Risiken einzugehen. Auf diese Weise erkennt der Sammelband auch an, dass Forschungsprozesse Praktiken der Erkundung mit offenem Ausgang sind, womit auch das Scheitern eine selbstverständlich eintretende Möglichkeit von Forschungsprozessen und etwas Produktives ist. Der Sammelband ist somit eine Publikation, die die aktuellen Debatten um Methoden in der Geographie im Kontext von cultural turn, postmodern identities, othering, positionality und research ethics widerspiegelt, kreativ umsetzt und methodische Innovation innerhalb der geographischen Methodenanwendung fördert.

Der Sammelband ist nach einer prägnanten Einleitung zu den Zielen und dem Zielpublikum des Buches sowie zur Einbettung des Begriffs Kreativität dem modellhaft linearen Forschungsprozess entsprechend in drei Teile gegliedert: Part I: Preparing for research, Part II: Creative and innovative methods, Part III: Handling creative methods.

Die Beiträge des ersten Teils besprechen Reflexivität, Positionalität und autoethnographische Praktiken (John Horton), diskutieren ob und wie die Erforschung des „Eigenen“ und des „Fremden“ überhaupt möglich ist (Chris Philo, Louise Boyle, Mark Lucherini), welche ethischen Überlegungen notwendig sind (Sarah Marie Hall, Gemma Sou, Laura Pottinger) und was Kreativität innerhalb von Forschungsprozessen bedeuten kann (Tracy Hayes). Während in Publi- 
kationen der Anfangsjahre diese Themen, wenn überhaupt, nur am Rande mitbesprochen wurden, verweist dieser Teil 1 des Sammelbandes auf die umfänglich etablierte Perspektive, dass Forschung situativ ist und damit notwendigerweise reflektiert werden muss. Dies ist nicht etwas, was nach einer Forschung noch gemacht werden kann, sondern vor der Forschung konsequent Beachtung finden muss.

Die Beiträge des zweiten Teils sind in fünf Sektionen unterteilt: Section I: Using Images and Visualisation Techniques, Section II: Multisensory and Embodied Approaches, Section III: Oral and Written Methods, Section IV: Using Technologies, Section V: Using and Producing Secondary Data. Mit den präsentierten visuellen, verbalen, körperbezogenen und technologiebasierten Methoden sowie deren Speicherung und Weiternutzung ist das Forschungsziel verbunden, alle Forschungsteilnehmenden in den Erhebungs- und Auswertungsprozess einzubinden. Damit werden Methoden bzw. methodische Vorgehen präsentiert, bei denen Daten nicht ,von jemandem“ „über jemanden“ erhoben, sondern Daten durch Interaktion im Forschungsprozess ko-kreiert werden.

Diese Ko-Konstruktion wird am Beispiel von Fotos (Noora Pyyry, Markus Hilander, Sirpa Tani), Moodboards (Jo Hickman-Dunne, Helena Pimlott-Wilson), Karten (Amy Mulvenna, Chris Perkins) und Zeichnungen wie beispielsweise Graffitis (Robin Kearns, Kyle Eggleton, Annie van der Plas, Tara Colemann) vorgestellt. Weiterhin beschäftigt sich der zweite Teil mit Sound (Geraint Rhys Whittaker, Kimberley Peters), Mobile Methods (Mark Riley, Jennifer Turner, Sam Hayes, Kimberley Peters, Morag Rose, Jane Samuels) und mit dem künstlerischen Gestalten und Performen (Katherine Harrison, Cassie Ogden, Sofie Narbed). Mit dieser Ausweitung des (geographischen) Forschungsinteresses geht jedoch die Arbeit am Erzählten und Schriftlichem nicht verloren. Zugang zu Literatur (Nichola Harmer, Gabriel Eshun, Clare Madge) wird ebenso besprochen wie zu Musiktexten (Geraint Rhys Whittaker, Kimberley Peters) und Biographien (Laura Fenton, Signe Ravn).

Der Einbezug digitaler Tools in die Erhebung und Auswertung von Forschungsdaten ist eine logische Konsequenz der zunehmenden Digitalisierung. Smartphones und Apps (Mike Duggan, Jamie Halliwell, Samantha Wilkinson, Mark Holton) kommen im Sammelband ebenso zur Sprache wie Videospiele und (Dokumentar-)Filme (Phil Jones, Tess Osborne, Thomas Aneurin Smith, Ria Ann Dunkley). Die Speicherung von Daten und die Wiederverwendung von (sekundären) Forschungsdaten in anderen Forschungskontexten und von anderen Forschenden bringt Potenziale, aber auch gewisse Herausforderungen mit sich, mit denen sich Matthew F. Rech, Catherine Wilkinson, Nadia von Benzon, Rhi Smith, Francesca Church und Hilary Geoghegan zum Abschluss des zweitens Teils beschäftigen und praktische Empfehlungen geben.

Diese vorangehende Auflistung zeigt die Breite gegenwärtiger Methodenanwendung innerhalb der Geographie. Da- bei kann nicht übersehen werden, dass die Vielfalt an methodischen Zugängen auch zu einer Vielfalt an Daten führt. Hinzu kommt, dass die jeweilige Anpassung von Methoden an den Forschungskontext die bereits bestehenden wissenschaftlichen Ansprüche an Nachvollziehbarkeit und Transparenz nochmals erhöht. Die Auseinandersetzung mit folgenden Fragen prägt den dritten Teil und damit den Abschluss des Sammelbandes: Wie kann mit der Datenvielfalt umgegangen werden? (Nadia von Benzon, Kimberley O'Sullivan), Wie kann Nachvollziehbarkeit und Transparenz hergestellt werden? (Pearlin Teow, Mark Holton), Wie können kreative Forschungsprozesse und damit kreativ gewonnene Forschungsergebnisse präsentiert und legitimiert werden? (Catherine Wilkinson, Lorna Brookes, Matthew Benwell, Bethan Evans, Andy Davies, Bernie Carter, Greg Thomas, Sergio A. Silverio).

Neben der inhaltlichen Vielfalt eignet sich das Buch aufgrund der intuitiv gestalteten Optik, der stringenten Strukturierung und der knackig prägnant gehaltenen Beiträge - und Beitragssprache - als Handbuch für die ein- und fortführende akademische Methodenlehre. Ein durchdachtes Glossar am Ende des Sammelbandes untermauert diese Einschätzung. Abschließend soll betont werden, dass die Perspektive der Autor*innen auf Methoden und deren Anwendung in der Geographie einer unterstützenden Atmosphäre gleicht, die nicht durch die Präsentation und Einforderung strenger Forschungsdesigns abschreckend, sondern überaus motivierend wirkt. Ein solcher Zugang ist für die gegenwärtige Forschungslandschaft wichtig und für Nachwuchswissenschafter*innen wird hier ein willkommenes Zeichen gesetzt!

Haftungsausschluss. Publisher's note: Copernicus Publications remains neutral with regard to jurisdictional claims in published maps and institutional affiliations. 